\title{
In die Zukunft ohne Gesamtkonzept: Vorausberechnungen und ihre Probleme
}

\author{
Johannes Geyer
}

\section{Einleitung}

Mit Ende des 20. Jahrhunderts wurde mit einer Reihe von Reformen ein einschneidender Reformprozess in der gesetzlichen Rentenversicherung (GRV) in Gang gesetzt. Angesichts steigender Beitragssätze und einer relativ hohen Arbeitslosigkeit zielte man auf eine Stabilisierung des Beitragssatzes zur GRV und auf die Dämpfung seines mittelfristigen Anstiegs. Mit der Einführung des Riester-Faktors ab 2002 wurden in der Folge mehrere Rentenanpassungen gedämpft und das Rentenniveau gesenkt (Steffen 2013). Mit dem RV-Nachhaltigkeitsgesetz wurde ab 2005 zudem der sogenannte Nachhaltigkeitsfaktor in die Rentenanpassungsformel eingeführt. Dieser Faktor modifiziert die jährliche Rentenanpassung - vereinfacht ausgedrückt - entsprechend der Veränderung des Verhältnisses von Beitragsund Rentensumme. Dadurch wird in der Formel ein „selbststabilisierender Mechanismus" wirksam, der die Anpassungskosten (gegebenenfalls auch Gewinne) bei einer Veränderung von Beitrags- und Rentensumme zwischen den Rentnerinnen und Rentnern (Rentenanpassung) auf der einen und den Beitragszahlerinnen und Beitragszahlern auf der anderen Seite verteilt (Börsch-Supan 2007). ${ }^{1}$ Dieser Reformprozess gilt als „Paradigmenwechsel“, weil im Zuge dieses Prozesses das Ziel der Beitragssatzstabilität das bisher gültige Leistungsziel ersetzte (Schmähl 2011). Die parallel umgesetzte und staatlich geförderte Teilprivatisierung der Alterssicherung im sogenannten Drei-Säulen-Modell sollte die Altersvorsorge nicht nur nachhaltiger gestalten, sondern auf ein besseres Fundament stellen: Durch die

1 Der Nachhaltigkeitsfaktor führt beispielsweise dazu, dass bei Leistungsausweitungen in der GRV zukünftige Rentenanpassungen geringer ausfallen, also auch die Rentnerinnen und Rentner auf diese Weise einen relevanten Teil der Kosten finanzieren (z.B. bei der Mütterente 2014, siehe dazu Bach et al. 2018). 
Mischung aus Umlage und Kapitaldeckung sollten im Drei-Säulen-Modell die Risiken gestreut und den Versicherten die "Chancen“ des Kapitalmarkts erschlossen werden. ${ }^{2}$

Die Einführung der Riester-Rente liegt bereits 18 Jahre zurück und es konnten einige Erfahrungen mit dem Drei-Säulen-Modell in Deutschland gesammelt werden. Zugleich findet eine lebhafte Debatte um die Zukunft und die Rolle der gesetzlichen Rentenversicherung statt und auch um die Zukunft des Drei-Säulen-Modells. Insbesondere mit Blick auf die GRV wird in dieser Debatte auf Vorausberechnungen zurückgegriffen, die die Entwicklung von Beitragssatz und Rentenniveau für die kommenden Jahrzehnte abzuschätzen versuchen. Unterschiedliche Schätzmodelle kommen dabei zwar zu leicht unterschiedlichen Ergebnissen, aber im Grundsatz unterscheiden sich die vorausgeschätzten Verläufe von Beitragssatz und Rentenniveau unter geltendem Recht nicht. Problematisch ist allerdings, dass in der Regel keine umfassende und systematische Betrachtung der Entwicklung aller Komponenten des 3-Säulenmodells geschieht. So geraten wichtige Fragen aus dem Blick, u.a.: Versichert die private Rente dieselben Risiken wie die GRV? Welche Gesamtbeitragsleistung haben die Versicherten zu tragen? Wie hoch sind die durchschnittliche Bruttoverzinsung und ihre Varianz? Welches Nettorentenniveau wird erreicht? Wie entwickeln sich die ausgezahlten Renten, die aus unterschiedlichen Quellen stammen, über die gesamte Rentenbezugszeit?

\section{Der Stand der Entwicklung der Alterssicherung}

In den Jahren seit der Reform hat sich gezeigt, dass das Drei-Säulen-Modell in Deutschland erhebliche Schwächen aufweist. Diese Schwächen resultieren nicht zuletzt aus dem politisch defensiven Reformansatz zum Einstieg in dieses Modell, der im Nachhinein als konzeptioneller Fehler zu bewerten ist: obwohl die private Vorsorge zur Aufrechterhaltung des Lebensstandards im Alter notwendig wurde, vermied man, sie als obligatorische Vor-

2 Exemplarisch für das Bild eines verbesserten Gesamtversorgungsniveaus, das Einkommen aus der GRV und private Renten kombiniert, stehen regelmäßig die Modellrechnungen im Alterssicherungsbericht der Bundesregierung (Bundesregierung 2016). Unter relativ günstigen Annahmen zu Zinsen, Sparvolumen und Länge der Erwerbsbiografie wird dort ein Netto-Gesamtversorgungsniveau für typisierte Modellbiografien ausgewiesen, das für alle Fälle einen Anstieg des zukünftigen Versorgungsniveaus vorhersagt (eine kritische Diskussion der Annahmen findet sich z.B. bei Schäfer 2015). 
sorge einzuführen (z.B. Wagner 2012). Dies hatte nicht nur eine unzureichende Verbreitung und voraussehbare Selektionseffekte zur Folge, sondern auch, dass bis heute keine zuverlässigen Aussagen über das zusätzliche private Sparvolumen (vgl. z.B. Corneo u.a. 2009) getroffen werden können. ${ }^{3}$ Die Verbreitung der betrieblichen Altersvorsorge stagniert seit einigen Jahren bei rund $56 \%$ aller sozialversicherungspflichtig Beschäftigten, im Jahr 2001 lag ihre Verbreitung bei knapp 49\% (Riedmann et al. 2019). Zudem ist das Bild des Drei-Säulen-Modells insofern irreführend, als die Säulen in unterschiedlicher Weise zur Risikoabsicherung beitragen. Die GRV versichert im Wesentlichen das Risiko der Langlebigkeit, der Erwerbsminderung und des Todes (Hinterbliebenenrente). Darüber hinaus garantiert sie als „dynamische Rente“ eine Partizipation am Produktivitätsfortschritt. Die GRV stellt damit die umfassendste der drei Säulen dar. Dagegen decken die privaten Versicherungen häufig nur das Risiko der Langlebigkeit ab und sind in der Regel nicht oder schwächer indexiert als die GRV. ${ }^{4}$

In den kommenden Jahren werden die geburtenstarken Jahrgänge („Baby-Boomer") in Rente gehen und zu einer merklichen Verschiebung im Verhältnis von Personen, die Beiträge zahlen, zu Personen, die Renten beziehen, beitragen. Der 14. koordinierten Bevölkerungsvorausberechnung des Statistischen Bundesamtes zufolge wird der Altersquotient - definiert als das Verhältnis von Personen im Alter zwischen 20 und 66 Jahren zu allen Personen, die 67 oder älter sind - in der mittleren Fortschreibungsvariante (G2-L2-W2) von 0,31 (2019) bis Ende der 2030er Jahre auf knapp 0,47 ansteigen. Auch danach wird der Quotient nicht weiter absinken, sondern sich auf diesem hohen Niveau stabilisieren und ab 2050 langsam weiter an-

3 Es herrscht sogar eine gewisse Unklarheit darüber wer zum förderfähigen Personenkreis zählt und auf welche Grundgesamt Verbreitungsquoten der Riester-Rente bezogen werden sollten (vgl. Fasshauer und Toutaoui 2009 und Geyer 2012).

4 Fachinger und Künemund (2014) diskutieren die unterschiedlichen Anpassungsmechanismen der Produkte der staatlich geförderten privaten Altersvorsorge und der betrieblichen Altersvorsorge kritisch. Bei der Riester-Rente existiert eine Nominalwertgarantie, aber keine Pflicht zur Anpassung der Leistungen im Rentenverlauf. Bei privaten Lebensversicherungen gibt es in der Regel eine Überschussbeteiligung - ob Überschüsse anfallen und wie hoch diese ausfallen bleibt dabei unsicher. Bei der betrieblichen Altersvorsorge gelten die Vorschriften des Betriebsrentengesetzes (BetrAVG). Hier sind die Anpassungsregeln relativ stark ausdifferenziert und könne sich je nach Durchführungsweg, konkreter Zusage und Unternehmen unterscheiden, eine systematische Teilhabe an der gesamtwirtschaftlichen Entwicklung wird durch diese Regelungen nicht sichergestellt (vgl. dazu auch die Ausführungen in Fachinger et al. 2014). 
steigen (Destatis 2020). Die rein demografischen Kennzahlen zeigen unmittelbar zwar noch nicht, wie sich das tatsächliche Verhältnis von Beitragszahlenden und Rentenbeziehenden verändern wird, sie geben aber eine Orientierung für den zu erwartenden finanziellen Druck auf die GRV. Entsprechend zeigen die Vorausschätzungen der Bundesregierung im jährlich erscheinenden Rentenversicherungsbericht und unabhängige Studien (z.B. Börsch-Supan et al. 2016; BMAS 2016; Ehrentraut/Moog 2017; Werding, 2016, 2018; Geyer et al. 2019; Deutsche Bundesbank 2019) gleichermaßen einen starken Anstieg des Beitragssatzes in den kommenden Jahren auf. Viele Beiträge in dieser Debatte folgern daraus die Notwendigkeit einer weiteren Absenkung der Leistungen der GRV, vor allem durch eine weitere Absenkung des Rentenniveaus und eine Erhöhung des Rentenzugangsalters (z.B. Sachverständigenrat 2016; Deutsche Bundesbank 2019). Damit verbunden ist die Forderung, die private und betriebliche Altersvorsorge weiter zu stärken.

Zugleich ist die jüngere Debatte über die GRV auch davon gekennzeichnet, dass wieder über Niveausicherungsziele diskutiert wird (Blank 2017). Das Sicherungsniveau vor Steuern ist zwischen 2000 und 2018 von $52,9 \%$ auf 48,3\% zurückgegangen und es wird nach geltendem Recht ein weiterer starker Rückgang erwartet. Wichtige Faktoren, die diese negative Entwicklung bedingen, sind der sogenannte Riester-Faktor und der Nachhaltigkeitsfaktor in der Rentenanpassungsformel. Der $₫ 154$ Abs. 3 SGB VI hatte zwar eine Untergrenze des Rentenniveaus von $43 \%$ bis 2030 vorgesehen, ohne aber festzulegen was passieren sollte, wenn diese Grenze unterschritten würde. Inzwischen werden sogar wieder höhere Niveausicherungsgrenzen diskutiert. Mit dem "Gesamtkonzept zur Alterssicherung“ wurde 2016 von der damaligen Bundesministerin für Arbeit und Soziales Andrea Nahles ein eigenes Konzept zur Stabilisierung der GRV vorgelegt. Dieses sah eine mindestens anzustrebende langfristige Haltelinie von 46\%, wenn möglich sogar $48 \%$ vor. Zwar wurde dieses Konzept nicht umgesetzt; im Koalitionsvertrag von 2018 wurde dann aber eine Haltelinie von $48 \%$ bis 2025 vereinbart (CDU, CSU und SPD 2018) und mit dem RVLeistungsverbesserungs- und -Stabilisierungsgesetz auch verabschiedet. ${ }^{5}$ Offen ist bisher, wie sich eine derartige Sicherung der Untergrenze des Rentenniveaus im Rahmen des Drei-Säulen-Modells gewährleisten lässt.

5 Es bleibt dabei ungewiss, ob die Haltelinie beim Rentenniveau überhaupt greifen wird. Nach dem Rentenversicherungsbericht 2019 wird weder die Haltelinie beim Rentenniveau noch die des Beitragssatzes bis 2025 erreicht. Stattdessen greift die untere Haltelinie beim Beitragssatz, d.h. dieser hätte ohne Haltelinie nach geltendem Recht gesenkt werden müssen. 
Langfristig erfordert diese politische Zielsetzung eine stärkere Anhebung des Beitragssatzes zur GRV. Soll der Gesamtbeitrag zur Altersvorsorge einschließlich privater und betrieblicher Vorsorge nicht unverhältnismäßig ansteigen, ist damit eine Schwächung der beiden anderen Säulen impliziert. Schon bei dieser vereinfachten Betrachtung wird deutlich, dass eine isolierte Betrachtung der GRV im Drei-Säulen-Modell nicht sinnvoll ist.

Zwei aktuelle Entwicklungen haben noch einen äußerst günstigen Einfluss auf die Finanzlage und die Leistungen der GRV: die mit dem aktuell stabilen Altersquotienten verbundene sogenannte "demografische Pause“ und insbesondere die anhaltend günstige Beschäftigungsentwicklung bzw. der starke und lang anhaltende Anstieg der sozialversicherungspflichtigen Beschäftigung. Dies führte zu einer überraschend günstigen Entwicklung des Beitragssatzes der GRV (Abbildung 1) und zu einem höheren Sicherungsniveau vor Steuern als erwartet (Abbildung 2). Noch im Jahr 2008 ging die Bundesregierung im Rentenversicherungsbericht für das Jahr 2019 von einem Beitragssatz von $20 \%$ aus, das erwartete Rentenniveau lag zugleich deutlich unter $47 \%$. Tatsächlich konnte der Beitragssatz gesenkt werden und liegt seit 2018 bei 18,6\%, und das Rentenniveau bewegt sich seit 2014 nur wenig und liegt aktuell bei 48,2\%. Bemerkenswert ist diese Entwicklung auch deswegen, weil mit den verschiedenen Rentenpaketen erstmals seit langer Zeit wieder Leistungsausweitungen in der GRV vorgenommen worden sind. Dazu zählen die Leistungsverbesserungen für Mütter von Kindern, die vor 1992 geboren wurden („Mütterrente“), die temporäre Absenkung der Altersgrenze für eine abschlagsfreie Altersrente besonders langjährig Versicherter und Verbesserungen bei der Erwerbsminderungsrente. Ohne diese Leistungsausweitungen, hätte der Beitragssatz stärker gesenkt werden können. Gleichzeitig wären dann auch die Rentenanpassungen etwas höher ausgefallen und der Bundeszuschuss wäre geringer ausgefallen. 
Abbildung 1: Beitragssatz zur GRV - Vorausschätzungen im Rentenversicherungsbericht zwischen 2008 und 2018

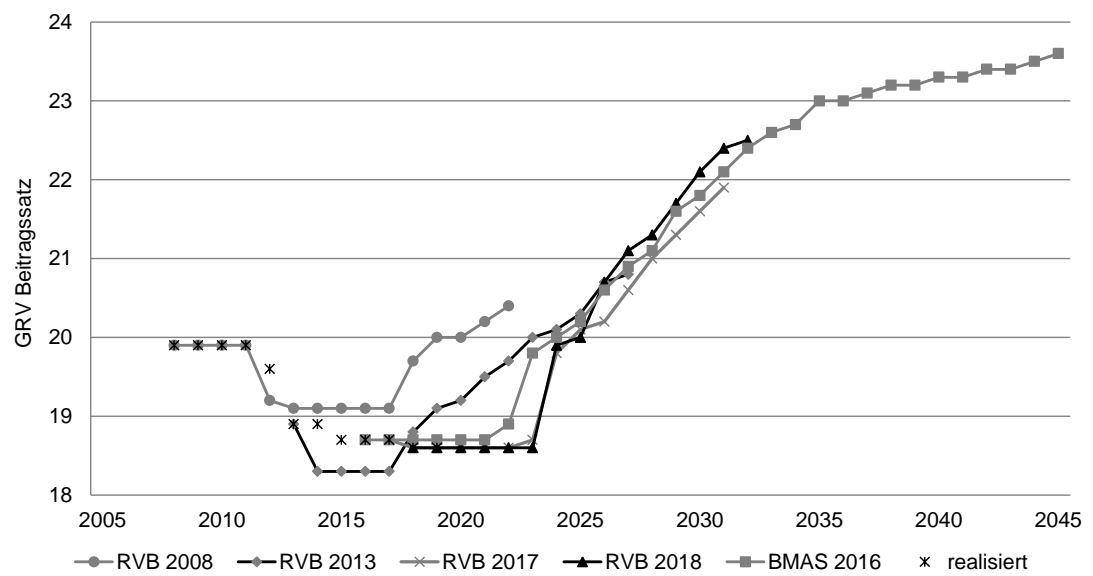

Quelle: Eigene Zusammenstellung auf Basis der Rentenversicherungsberichte 2008, 2013, 2017, 2018 und BMAS (2016)

Abbildung 2: Sicherungsniveau vor Steuern - Vorausschätzungen im Rentenversicherungsbericht zwischen 2008 und 2018

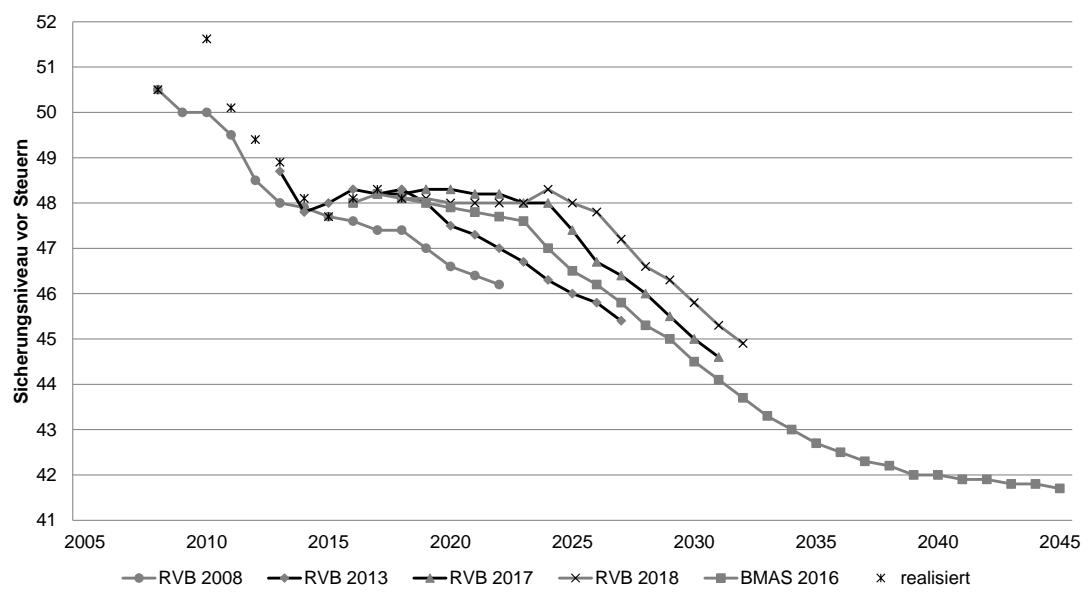

Quelle: Eigene Zusammenstellung auf Basis der Rentenversicherungsberichte 2008, 2013, 2017, 2018 und BMAS (2016) 
Da die geburtenstarken Jahrgänge nun in das Rentenalter vorrücken, wird sich diese Entwicklung absehbar nicht länger fortsetzen. Die Bundesregierung geht von einer relativen Stabilität der Rente noch bis zum Jahr 2025 aus. Die im Jahr 2018 formierte Kommission „Verlässlicher Generationenvertrag“ wird im Frühjahr 2020 Vorschläge für die Weiterentwicklung des Rentensystems nach 2025 vorlegen.

Welche Entwicklung unter den aktuellen Regelungen der GRV und mit den Annahmen der gängigen Fortschreibungsmodelle über die Arbeitsmarkt- und die demografische Entwicklung zu erwarten ist, wird im Folgenden kurz umrissen. Daran anschließend werden die wichtigsten rentenpolitischen Stellschrauben diskutiert, mit denen den Herausforderungen des demografischen Wandels nachhaltig begegnet werden könnte. Dabei wird auch herausgearbeitet, welche Probleme mit aktuellen Vorausberechnungen verbunden sind.

\section{Vorausschätzungen und Handlungsoptionen}

Eine wichtige Grundlage der Arbeit an tragfähigen Zukunftskonzepten ist die Vielzahl der Vorausschätzungen über die Finanzlage der GRV und das zu erwartende Sicherungsniveau, die in den letzten Jahren erschienen sind. So liefert der jährlich veröffentlichte Rentenversicherungsbericht der Bundesregierung eine mittelfristige Vorausschätzung der Entwicklung über die nächsten 15 Jahre. Daneben werden regelmäßig Vorausberechnungen mit einem längeren Fortschreibungshorizont, teilweise bis zum Jahr 2080, veröffentlicht (z.B. Deutsche Bundesbank 2016, 2019; Geyer et al. 2019; Ehrentraut/Moog 2017; Werding 2016, 2018; Börsch-Supan/Rausch 2018). Zwar unterscheiden sich die Studien im Detail, was die zugrunde liegenden Simulationsmodelle, ihre Datenbasis, den konkret simulierten Rechtsstand und einige weitere Annahmen betrifft; im Ergebnis schätzen sie aber einen relativ einheitlichen Trend.

Danach ist zu erwarten, dass der Beitragssatz bei Fortschreibung des geltenden Rechtsstandes zur GRV im Jahr 2030 zwischen 1,9 und 3,7 Prozentpunkten über dem heutigen Niveau liegen wird, im Mittel wird ein Anstieg von knapp drei Punkten vorausgeschätzt (Tabelle 1). Den Schätzungen zufolge wird das Rentenniveau im Schnitt ebenfalls knapp drei Punkte unter dem Niveau von 48,3\% des Jahres 2019 liegen. Und diese ne- 
gative Entwicklung wird sich fortsetzen, so dass für das Jahr 2045 ein Beitragssatz von mehr als $23 \%$ erwartet wird und - in den meisten Studien ein weiteres Absinken des Sicherungsniveaus auf unter $43 \%{ }^{6}$

Tabelle 1: Entwicklung von Beitragssatz und Sicherungsniveau vor Steuern der gesetzlichen Rentenversicherung in verschiedenen Vorausberechnungen

\begin{tabular}{|c|c|c|c|c|c|c|c|c|c|c|c|c|c|c|}
\hline \multirow[t]{2}{*}{ Jahr } & \multicolumn{4}{|c|}{$\begin{array}{l}\text { Börsch-Su- } \\
\text { pan und } \\
\text { Rausch } \\
(2018)\end{array}$} & \multicolumn{2}{|c|}{$\begin{array}{l}\text { Werding } \\
(2016)\end{array}$} & \multicolumn{2}{|c|}{$\begin{array}{l}\text { Werding } \\
(2018)\end{array}$} & \multicolumn{2}{|c|}{$\begin{array}{c}\text { Deutsche } \\
\text { Bundes- } \\
\text { bank } \\
(2016)\end{array}$} & \multicolumn{2}{|c|}{$\begin{array}{c}\text { Buslei et al. } \\
\text { (2019) }\end{array}$} & \multicolumn{2}{|c|}{$\begin{array}{c}\text { Burret und } \\
\text { Moog } \\
(2019)\end{array}$} \\
\hline & BS & SN & BS & SN & BS & SN & BS & SN & BS & SN & BS & SN & BS & SN \\
\hline 2030 & 21,8 & 44,5 & 21,6 & 45 & 20,5 & 47,1 & 20,7 & 46,3 & 21,8 & 44,1 & 21,5 & 46,2 & 22,3 & 46 \\
\hline 2040 & 23,3 & 42 & & & 22,0 & 44,8 & 22,5 & 43,8 & & 41,8 & & & 24,3 & 42,8 \\
\hline 2045 & 23,6 & 41,7 & 23,2 & 42,3 & 22,5 & 44,2 & 23,2 & 43,2 & & 41,7 & 23,6 & 43,2 & & \\
\hline 2050 & & & & 42 & 23,1 & 43,4 & 23,9 & 42,3 & & 41,4 & & & 25 & 41,7 \\
\hline 2060 & & & 24 & 41,1 & 24,0 & 42,0 & 25,4 & 40,5 & $>24$ & 40,4 & & & 25,7 & 40,9 \\
\hline 2070 & & & & & 24,2 & 41,6 & & & & & & & 25,9 & 40,9 \\
\hline 2080 & & & & & 24,3 & 41,3 & 26,8 & 38,8 & & & & & & \\
\hline
\end{tabular}

Anmerkungen: BS - Beitragssatz. SN - Sicherungsniveau vor Steuern. In den jeweiligen Simulationen werden neben Unterschieden in den Modellen, der Datengrundlage und den demografischen Annahmen unterschiedliche Rechtszustände unterstellt. Quelle: Eigene Zusammenstellung aus den oben zitierten Quellen und Kaltenborn (2019).

So einheitlich die Ergebnisse der Studien sind, so unterschiedlich sind die daraus abgeleiteten Empfehlungen, wie den Herausforderungen des demografischen Wandels am besten zu begegnen wäre. Wie in vorangegangenen Abschnitt kurz illustriert, wird auf der einen Seite die Stabilisierung der Leistungen der GRV als zentrales Ziel hervorgehoben, und auf der anderen Seite wird an der Stoßrichtung der Reformen, die Beitragsstabilität zu sichern, festgehalten und auf den Ausbau der privaten Vorsorge gedrungen.

Die Schwierigkeit für die rentenpolitische Debatte liegt darin, dass diese Vorschläge und ihre künftigen Wirkungen oft nur schwer vergleichbar sind, weil eine systematische vergleichende Betrachtung der Belastungen

6 Ein ausführlicher Vergleich verschiedener aktueller Studien, die die langfristigen Auswirkungen des demografischen Wandels untersuchen, findet sich in Kaltenborn 2019. 
bzw. Kosten und Leistungen fehlt, und der Fokus teils auf die GRV beschränkt bleibt. Dies soll kurz anhand der typischen Handlungsstrategien erläutert werden.

Die zentralen Handlungsoptionen bei dem umlagefinanzierten deutschen Rentensystem, das der Beitragsäquivalenz einen großen Stellenwert einräumt, sind bekannt. ${ }^{7}$ Entscheidend für die präferierte Politik ist dabei, ob versucht werden soll, die GRV als tragende Säule der Alterssicherung zu stärken oder ob die Richtung der Beitragssatzstabilisierung bei sinkendem Niveau weiter verfolgt wird. Wenn man letztere Alternative präferiert, sollte man auch die Ausgestaltung der privaten Vorsorge thematisieren, da die dort gewährten Leistungen und die entsprechenden Belastungen dann als integraler Bestandteil des Alterssicherungssystems interpretiert werden müssen. Im Folgenden sollen kurz zwei Aspekte der GRV diskutiert werden, die dabei immer wieder Gegenstand von Kontroversen sind: (1) die Höhe der Beiträge und der Steuerzuschüsse und (2) das Rentenniveau.

\subsection{Parameter und Stellschrauben der Rentenpolitik: Beitragssatz \& Steuerzuschuss}

Der Beitragssatz ist eine naheliegende Stellschraube, um die Einnahmen zu erhöhen und Leistungen zu finanzieren. Wie oben erläutert, steigt der Beitragssatz zur GRV in der mittleren Frist bereits nach heutigem Recht mittelfristig relativ stark an. Modelle, in denen das Leistungsniveau entgegen dem geltenden Recht über 2025 hinaus stabilisieren wird, schätzen einen nochmal höheren Finanzbedarf, also höhere Beitragssätze (Ehrentraut/Moog 2017; Werding 2018; Börsch-Supan/Rausch 2018). Neben den Beiträgen steigen auch die Steuermittel. Die GRV erhält Steuermittel vor allem in der Form der Bundeszuschüsse und als Beiträge für Kindererziehungszeiten. Theoretisch sollen die Bundesmittel zur Finanzierung sogenannter versicherungsfremder Leistungen genutzt werden, allerdings ist das genaue Volumen der nicht beitragsgedeckten Leistungen nicht bekannt (Deutsche Rentenversicherung 2019). ${ }^{8}$ Faktisch ist die Zweckbindung nicht relevant für die Ausgestaltung und Fortschreibung der Bundes-

7 Es handelt sich hier im Wesentlichen um das Leistungsniveau, die Höhe des Beitragssatzes, das Regelrenteneintrittsalters und die Rolle des Bundeszuschusses.

8 Die Wissenschaftlichen Dienste schreiben in einem Gutachten von 2016: „Die Einschätzung von Wissenschaft und Politik ist uneinheitlich, je nachdem, welches Ziel durch die Beitragszahlung verfolgt werden soll." (Wissenschaftliche Dienste 
zuschüsse (Wissenschaftliche Dienste 2018). Beispielhaft kann man in diesem Zusammenhang auch die Mütterrente I und II $^{9}$ nennen, die nur zu einem Teil aus Steuermitteln finanziert wird, obwohl es sich um Beiträge für Kindererziehungszeiten handelt. Die gesamten Bundesmittel lagen im Jahr 2018 bei fast 95 Milliarden Euro, rund 30\% der Einnahmen der GRV (Deutsche Rentenversicherung Bund 2019). Würde man diesen Steuerzuschuss zusätzlich ausweiten, um das Leistungsniveau der GRV zu erhalten und den Beitragssatz zu stabilisieren ${ }^{10}$, würden sich systematische Fragen stellen, da nicht versicherte Gruppen wie die Beamten oder Teile der Selbständigen über Steuern stärker belastet würden. Börsch-Supan und Rausch (2018) schätzen beispielsweise, dass der zusätzliche Finanzbedarf einer doppelten Haltelinie (Beitragssatz und Rentenniveau) über 2025 hinaus bis 2030 eine Erhöhung der Mehrwertsteuer um drei Prozentpunkte erfordern würde. Langfristig wären es sogar sechs oder mehr Punkte.

In den GRV-Modellen ist es naheliegend, diese Belastungen in Form von höheren Beiträgen und Steuerzuschüssen bei einer Variation der Politik explizit auszuweisen. ${ }^{11}$ Theoretisch wäre es konsequent, hier auch den privaten Finanzierungsbedarf als Teil der Beitragsbelastung auszuweisen, der als notwendig für die Lebensstandardsicherung sowie zur Absicherung anderer Risiken (Erwerbsminderung, Hinterbliebenenschutz) angesehen wird. Das wird aber in der Regel nicht gemacht, so dass die Gesamtbelastung unterschätzt wird und im Vergleich zum Drei-Säulen-Modell die Belastungen durch steigende Beitragssätze in der GRV erheblich überzeichnet werden (z.B. Börsch-Supan/Rausch 2018; Werding 2018). ${ }^{12}$ So werden dann zwar diese Kosten quantifiziert, aber nicht gegengerechnet, dass die

2016). Auch Meinhardt (2018) weist auf die unterschiedlichen Definitionen hin, er schätzt ein Volumen je nach Abgrenzung der Leistungen zwischen 26 und gut 48 Milliarden Euro für 2016.

9 Nach dem RV-Leistungsverbesserungsgesetz (2014) und dem Leistungsverbesserungs- und -Stabilisierungsgesetz (2018). Siehe dazu Bach et. al 2014 und Bach u. a. 2018.

10 So ein Vorschlag war Teil des „Gesamtkonzeptes zur Alterssicherung“ (BMAS 2016). Der Vorschlag sah die Einführung eines „Demografiezuschusses“ vor. In dieser Perspektive wird die Stabilisierung der GRV als gesamtgesellschaftliche Aufgabe verstanden.

11 Den zusätzlichen Finanzbedarf der GRV in Form von Mehrwertsteuerpunkten auszuweisen wie bei Börsch-Supan und Rausch (2018) ist willkürlich gewählt und dient den Autoren nur zu Illustration des zusätzlichen Finanzvolumens. Man hätte das auch an der Einkommensteuer oder am BIP ausdrücken können oder in Form von Beitragsentlastungen bei der privaten Vorsorge.

12 Umgekehrt sollte bei der GRV-Beitragsbelastung auch der gesamte Beitragssatz als Belastung definiert werden. Es stimmt zwar, dass der Beitragssatz paritätisch 
Menschen bei einer Leistungsstabilisierung oder sogar Ausweitung der Leistungen in der GRV dann weniger in die private Altersvorsorge investieren müssten. Konsequent wäre es zudem darauf hinzuweisen, dass der Verbreitungsgrad privater Versicherung deutlich hinter der Verbreitung der GRV zurückbleibt. Aufgrund der Notwendigkeit einen Kapitalstock erst bilden zu müssen, würde man zudem selbst bei einem staatlich verordneten Obligatorium erst langfristig ein vergleichbares substanzielles Sicherungsniveaus erreichen. Außerdem wäre es hier zentral zu erklären, dass das Risiko der Erwerbsminderung und vermutlich auch Hinterbliebenenleistungen im Beitragssatz zur privaten Versicherung nicht abgedeckt werden.

\subsection{Parameter und Stellschrauben der Rentenpolitik: Rentenniveau \& Altersgrenzen}

Auch beim Leistungsniveau stellen sich beim Vergleich unterschiedlich gemischter Systeme einige grundlegende Fragen. Das gängige Rentenniveau ist ein abstraktes Konzept, das das Verhältnis der Standardrente (45 Entgeltpunkte) und des Durchschnittsentgelts der Versicherten misst. Es ist eine wichtige Kenngröße oder Maßzahl der Leistungsfähigkeit der umlagefinanzierten lohnorientierten GRV und erlaubt einen Vergleich ihrer Leistungen über die Zeit. In der rentenpolitischen Debatte ist es dabei üblich, über das sogenannte Sicherungsniveau vor Steuern zu sprechen, da ein einheitliches Nettorentenniveau aufgrund des Übergangs zur nachgelagerten Besteuerung nicht berechnet werden kann. Das Sicherungsniveau vor Steuern berücksichtigt nur die Belastungen durch die Sozialbeiträge und private Vorsorge. ${ }^{13}$

In der rentenpolitischen Diskussion erhält die zukünftige Entwicklung des Rentenniveaus eine hohe Bedeutung. Seine Senkung steht stellvertretend entweder für eine finanziell nachhaltige Rentenpolitik oder für eine zunehmend prekäre Einkommenssituation für eine zunehmende Zahl von Rentenbeziehenden. Wie oben erläutert, wird inzwischen eine Untergrenze beim Rentenniveau von vielen Seiten in der Rentendebatte unterstützt. Hier zeigen sich ähnlich Schwierigkeiten beim Vergleich eines Rentenni-

von Arbeitgebenden und Arbeitnehmenden finanziert wird, aber im Ergebnis handelt es sich dabei um Lohnkosten. Kurzfristig kann die Inzidenz davon abweichen, da eine Anpassung des Beitragssatzes unmittelbar Löhne erhöht bzw. senkt.

13 Zur Diskussion der verschiedenen Niveaugrößen und der jüngsten Neudefinition des Sicherungsniveaus vor Steuern, siehe Steffen (2019). 
veaus, das sich ausschließlich auf die GRV bezieht, und eines Sicherungsniveaus, das auch andere Quellen berücksichtigt. Es gelten hier zudem die Argumente wie beim Beitragssatz bezüglich der versicherten Risiken: weil die unterschiedlichen Altersvorsorgevarianten unterschiedliche Risiken versichern, ist ein Gesamtversorgungsniveau, das sich aus GRV, RiesterRente und sonstiger privater Altersvorsorge zusammensetzt, nicht so aussagekräftig wie es scheint. Die privaten Versicherungen decken häufig nur das Risiko der Langlebigkeit ab und versichern nicht gegen Erwerbsminderung oder den Tod. Zudem stellt sich die Frage wie sich das aus unterschiedlichen Quellen zusammengesetzte Leistungsniveau über die Zeit entwickelt, also ob und nach welchen Kriterien private Rentenleistungen in der Auszahlungsphase dynamisiert werden (vgl. dazu Fußnote 4).

In jüngeren Veröffentlichungen wird ein weiterer Vorschlag zur Stabilisierung des Rentenniveaus formuliert, der ohne zusätzliche Ausgaben in der GRV auskommt. Dabei wird die Erwerbsbiografie zur Berechnung der Standardrente mit der Anhebung der Altersgrenzen verlängert (z.B. Sachverständigenrat zur Begutachtung der gesamtwirtschaftlichen Entwicklung 2016; Werding 2018; Deutsche Bundesbank 2019). Wenn sich die Biografie der Standardrente (45 Jahre Durchschnittsentgelt) entsprechend um zwei Jahre erhöht, würde das Rentenniveau mechanisch steigen. Allerdings tritt dieser Effekt ein, weil sich die Basis des Vergleichs verändert, obwohl sich an den objektiven Verhältnissen nichts verändert hat. ${ }^{14}$ Man kann das tun, aber es hat eher einen normativen Charakter und wenig Erkenntniswert. Werding (2018) simuliert diesen Effekt und lässt die Altersgrenze auch über das Alter 67 hinaus - gekoppelt an die steigende Lebenserwartung steigen. Die Altersgrenze wächst dabei zwischen 1,2 und 1,5 Monaten pro Jahr. Er zeigt, dass unter diesen Annahmen einer ansteigenden Standardbiografie und mit privater Vorsorge das kombinierte Sicherungsniveau langfristig stabil gehalten werden kann. Spiegelbildlich wird der Anstieg des Beitragssatzes zur GRV gedämpft. Wie hoch die kombinierten Belastungen aus öffentlicher Vorsorge und privater Absicherung sind, wird nicht gezeigt. Zudem wird das kombinierte Rentenniveau nur für die jeweilige Zugangskohrte ausgewiesen - für die ist es stabil. Da sich aber die Definition der Standardbiografie mit der Zeit ändert, sinkt das Rentenniveau für den Rentenbestand kontinuierlich ab.

14 Das ist übrigens ein Grund für das relativ hohe Gesamtversorgungsniveau im Alterssicherungsbericht. Die jüngeren Biografien arbeiten und sparen über einen längeren Zeitraum, weil angenommen wird, dass sie später in Rente gehen (Schäfer 2015). 


\section{Diskussion}

Die aktuelle rentenpolitische Debatte ist unübersichtlich. Bis 2025 gelten doppelte Haltelinien für Niveau und Beitragssatz, danach bleibt viel zu entscheiden. Das deutsche Alterssicherungssystem ist geprägt vom unvollendeten Übergang zum Drei-Säulen-Modell. So wurde das Leistungsniveau der GRV gesenkt, ohne adäquaten Ersatz in der zweiten und dritten Säule sicherzustellen. Das betrifft neben der unzureichenden Verbreitung der privaten Vorsorge auch die bleibenden Lücken bei der Versicherung des Risikos der Erwerbsminderung und bei der Hinterbliebenenversorgung. Die gleichsam entstehende Beitragsbelastung in der zweiten und dritten Säule wird systematisch ausgeblendet. Gleichzeitig ist in naher Zukunft mit steigenden GRV-Beitragssätzen und einem weiteren Absenken des Rentenniveaus zu rechnen. Eine Stabilisierung oder auch eine weitere Absenkung des Rentenniveaus sollte im Kontext des anzustrebenden Alterssicherungssystems diskutiert werden.

Das gilt auch für die vielen verschiedenen Modellrechnungen, die Langfristprognosen zur GRV erstellen. Hier liegt der Fokus ausschließlich auf der GRV. Die Modellierung der privaten ergänzenden Vorsorge ist in der Regel einfach gehalten und überzeichnet ihren positiven Beitrag zur Alterssicherung systematisch. Bei der Modellierung unterschiedlicher rentenpolitischer Szenarien in der GRV ist es naheliegend die Belastungen in Form von höheren Beiträgen und Steuerzuschüssen explizit auszuweisen. Allerdings fehlt in der Regel bei diesen Berechnungen eine explizite Modellierung der Belastungen in der zweiten und dritten Säule. Das führt in der Konsequenz zu einer Überschätzung der finanziellen Belastungen durch steigende GRV-Beitragssätze und Steuern. Und auf der Leistungsseite werden die privaten Versicherungen eher zu günstig dargestellt. Es wäre wichtig zu erklären, dass das Risiko der Erwerbsminderung und in der Regel auch Hinterbliebenenleistungen im Beitragssatz zur privaten Versicherung nicht abgedeckt wird und die Niveaulücke deswegen auch nur teilweise geschlossen werden kann. Zudem wird dabei systematisch ignoriert, dass der Verbreitungsgrad privater Versicherung deutlich hinter der Verbreitung der GRV zurückbleibt und ein Kapitalstock für einen großen Teil der Bevölkerung erst aufgebaut werden muss.

Gerade weil ein Gesamtkonzept zur Alterssicherung über 2025 hinaus fehlt, wäre es wünschenswert bei der zukünftigen Modellierung der langfristigen Entwicklung der Alterssicherung die unterschiedlichen Säulen auch entsprechend zu berücksichtigen. 


\section{Literatur}

Bach, S./Buslei, H./Coppola, M./Haan, P./Rausch, J. (2014): Die Verteilungswirkungen der Mütterrente, in: DIW Wochenbericht 81 (20), S. 447-456

Bach, S./Buslei, H./Harnisch, M. (2018): Die Mütterrente II kommt vor allem Rentnerinnen mit geringen und mittleren Einkommen zugute, in: DIW Wochenbericht 85 (28), S. 613-22

Blank, F. (2017): Das Rentenniveau in der Diskussion. Policy Brief WSI 13, Düsseldorf

Bundesministerium für Arbeit und Soziales (BMAS) (2016): Gesamtkonzept zur Alterssicherung, Berlin

Börsch-Supan, A. (2007): Über Selbststabilisierende Rentensysteme. MEA Discussion Paper Series 133-2007, https:/www.mpisoc.mpg.de/fileadmin/user_upload /datapool/publications/cggcuv2xvve3ual7_133-2007.pdf (aufgerufen am 03.02.2020)

Börsch-Supan, A./Rausch, J. (2018): Die Kosten Der Doppelten Haltelinie. In: ifo Schnelldienst 71 (09), S. 23-30

Börsch-Supan, A./Bucher-Koenen, T./Rausch, J. (2016): Szenarien für eine nachhaltige Finanzierung der Gesetzlichen Rentenversicherung, in: ifo Schnelldienst 69 (18), S. 31-40

Bundesregierung (2016): Alterssicherungsbericht 2016. Ergänzender Bericht der Bundesregierung zum Rentenversicherungsbericht 2016

Burret, H./Moog, S. (2019): Einbeziehung von Beamtinnen in die gesetzliche Rentenversicherung. WISODirekt 20, Bonn.

CDU/CSU/SPD (2018): Ein neuer Aufbruch für Europa. Eine neue Dynamik für Deutschland. Ein neuer Zusammenhalt für unser Land. Koalitionsvertrag zwischen CDU, CSU und SPD. 19. Legislaturperiode

Corneo, G./Keese, M./Schröder, C. (2009): The Riester Scheme and Private Savings: An Empirical Analysis based on the German SOEP, in: Schmollers Jahrbuch: Journal of Applied Social Science Studies / Zeitschrift für Wirtschafts- und Sozialwissenschaften 129 (2): S. 321-332

Destatis (2020): Vorausberechneter Bevölkerungsstand: Deutschland, Stichtag, Varianten der Bevölkerungsvorausberechnung, Geschlecht, Altersjahre, Genesisonline Datenbank, Code 12421-0002, https://www-genesis.destatis.de/genesis/o nline (abgerufen am 03.02.2020)

Deutsche Bundesbank (2016): Exkurs: Zur längerfristigen Entwicklung der Alterssicherung, in: Monatsbericht August, S. 69-77

Deutsche Bundesbank (2019): Langfristige Perspektiven der gesetzlichen Rentenversicherung. Monatsbericht Oktober, S. 55-82

Deutsche Rentenversicherung Bund (2019): Nicht beitragsgedeckte Leistungen und Bundeszuschüsse 2017, Berlin

Deutsche Rentenversicherung Bund (2019): Rentenversicherung in Zeitreihen. DRV-Schriften 22, Berlin 
Ehrentraut, O. (2007): Alterssicherung in Deutschland - das Zusammenspiel der drei Säulen, in: Deutsche Rentenversicherung 62 (8-9), S. 576-89

Ehrentraut, O./Moog, S. (2017): Zukunft der gesetzlichen Rentenversicherung: Möglichkeiten und Grenzen ausgewählter Reformvorschläge, Hans-Böckler-Stiftung Study 345, Düsseldorf

Fachinger, U./Künemund, H. (2014): Stabilität des Versorgungsniveaus nach dem Paradigmenwechsel, in: Vierteljahrshefte zur Wirtschaftsforschung 83 (2), S. 3347

Fachinger, U./Künemund, H./Unger, K./Koch, H./ Schmähl, W./Laguna, E. (2014): Die Dynamisierung von Alterseinkommen - Chancen und Risiken eines neuen Mischungsverhältnisses staatlicher, betrieblicher und privater Alterssicherung: Income Dynamics in Old Age - IDA. Herausgegeben von Deutsche Rentenversicherung Bund, DRV-Schriften 104, Berlin

Fasshauer, S./Toutaoui, N. (2009): Die Anzahl des förderberechtigten Personenkreises der Riester-Rente - eine Annäherung. Deutsche Rentenversicherung 64 (6), S. 478-486

Geyer, J. (2012): Riester-Rente und Niedrigeinkommen: was sagen die Daten?, in: Vierteljahrshefte zur Wirtschaftsforschung 81 (2), S. 165-80

Geyer, J./Buslei, H./Gallego-Granados, P./Haan, P. (2019): Anstieg der Altersarmut in Deutschland: Wie wirken verschiedene Rentenreformen? Gütersloh

Kaltenborn, B. (2019): Wirkungen des anstehenden demographischen Wandels auf die sozialen Sicherungssysteme in Deutschland, in: Opielka, M. (Hrsg): Zukunftslabor Schleswig-Holstein - Demographie und Digitalisierung \#ZLabSH, Norderstedt, S. 24-110

Meinhardt, V. (2018): Versicherungsfremde Leistungen der Sozialversicherung, IMK Study 60, Düsseldorf

Riedmann, A./Thorsten H./Günther D./Walther, S./Blancke, S. (2019): Trägerbefragung zur Verbreitung der betrieblichen Altersversorgung (BAV 2017), Endbericht (Forschungsbericht FB523 des Bundesministerium für Arbeit und Soziales), München

Sachverständigenrat zur Begutachtung der gesamtwirtschaftlichen Entwicklung (2016): Zeit für Reformen: Jahresgutachten 2016/17, Paderborn

Schäfer, I. (2015): Die Illusion von der Lebensstandardsicherung - Eine Analyse der Leistungsfähigkeit des „Drei-Säulen-Modells“, Bremen

Schmähl, W. (2011): Von der Ergänzung der gesetzlichen Rentenversicherung zu deren partiellen Ersatz: Ziele, Entscheidungen sowie sozial- und verteilungspolitische Wirkungen - Zur Entwicklung von der Mitte der 1990er Jahre bis 2009, in: Eichenhofer E./Rische, H./Schmähl, W. (Hrsg.): Handbuch der deutschen Rentenversicherung SGB VI, Köln, S. 169-249

Steffen, J. (2013): Wirkungsanalyse der, Riester'-Treppe, http://www.portal-sozialpo litik.de/uploads/sopo/pdf/2013/2013-04-03-Riester_Treppe_PS.pdf (abgerufen am 03.02.2020) 
Steffen, J. (2018): Neue Berechnung des Rentenniveaus im Rahmen des Gesetzes über Leistungsverbesserungen und Stabilisierung in der gesetzlichen Rentenversicherung, http:/www.portal-sozialpolitik.de/uploads/sopo/pdf/2018/2018-07-30 _Neuberechnung_Rentenniveau_PS.pdf (abgerufen am 30.02.2020)

Wagner, G. G. (2012): Anmerkungen zur Geschichte und zur aktuellen Diskussion der Riester-Rente, in: Vierteljahrshefte zur Wirtschaftsforschung 81 (2), S. 27-33

Werding, M. (2016): Rentenfinanzierung im demographischen Wandel: Tragfähigkeitsprobleme und Handlungsoptionen. Sachverständigenrat zur Begutachtung der gesamtwirtschaftlichen Entwicklung. Arbeitspapier 05, Wiesbaden

Werding, M. (2018): Demographischer Wandel, soziale Sicherung und öffentliche Finanzen: Langfristige Auswirkungen und aktuelle Herausforderungen, Gütersloh

Wissenschaftliche Dienste (2016): Sachstand: Nicht beitragsgedeckte versicherungsfremde Leistungen der gesetzlichen Rentenversicherung als Ausdruck des Solidarprinzips, WD 6-3000-085/16, https://www.bundestag.de/blob/437624/634ba ef7575ec97bc241976afb1168e4/wd-6-085-16-pdf-data.pdf (abgerufen am 30.02.2020)

Wissenschaftliche Dienste (2018): Fortschreibung der Bundeszuschüsse zur gesetzlichen Rentenversicherung, WD 6-3000-094/18. https:/www.bundestag.de/reso urce/blob/580942/819a1ff8f2cb4ba6ed2e7221f28f694d/wd-6-094-18-pdf-data.pdf (abgerufen am 30.02.2020) 